\title{
Intrinsic angular investigations on metal evaporated tapes
}

\author{
E.O. Samwel ${ }^{*}$, D.B. Bijl, J.C. Lodder, Th.J.A. Popma \\ MESA Research Institute, University of Twente, P.O. Box 217, 7500 AE Enschede, The Netherlands
}

\begin{abstract}
Demagnetisation compensated torque- and angle-dependent remanence measurements are presented on commercial and experimental metal evaporated tapes. The latter have been prepared in our laboratory on a mini-roll coater system. From these results the easy axes have been determined.
\end{abstract}

\section{Introduction}

With the growing interest in thin-film media with oblique anisotropy directions, the importance of angular investigation methods has also increased. Both the well known torque measurements [1] and angle dependent remanence (ADR) curves [2] are suitable for detecting important parameters such as the easy axis direction.

ADR curves provide information not only on the global easy axis but also on 'local' easy axes. This is very useful when investigating double-layer media, where each layer has its own easy axis.

\section{The demagnetising field}

Both torque and ADR measurements are strongly influenced by the demagnetising field. Due to the oblique anisotropy direction, in almost all situations, a magnetisation component perpendicular to the sample surface is present, which causes a demagnetising field that influences the field inside the material. Therefore the results obtained by 'standard' measurements present information on the easy axis that results from the summation of the intrinsic anisotropy direction and the demagnetising field. The intrinsic anisotropy direction is determined mainly by the inclination of the columns and their shape anisotropy in obliquely evaporated media. If compensated for the demagnetising field, the measured anisotropy direction should reveal information on the (average) direction of these columns. In a recording situation the net magnetisation is always zero, so that bit patterns are always written in the absence of a global demagnetising field. This means that the magnetisation direction in these bits is determined only

\footnotetext{
* Corresponding author. Fax: +31-53-430-9547; email: samwel@eltn.utwente.nl.
}

by the intrinsic easy axis and the stray field of neighbouring bits.

In principle, it is possible to compensate torque measurements for the demagnetising field after the measurement has been done by subtracting the 'sheet demagnetisation anisotropy' vector. For torque measurements performed in a torque magnetometer the magnetisation component perpendicular to the sample surface, which is the origin of the demagnetising field, is not known. It can only be calculated by assuming complete alignment of the magnetisation with the applied field, which is only the case at infinite field. At lower fields extrapolation to an infinite field is necessary.

Torque curves derived from biaxial VSM measurements do not have this disadvantage. Because all components of the magnetisation vector are known, the demagnetising field can be calculated more easily. A disadvantage is that torque measurements in a VSM have lower sensitivity. This is because the torque is calculated from the magnetisation component perpendicular to the field, which becomes very small at high fields. For ME tape media the sensitivity is sufficient however.

Due to the changing $M_{y}$ component, the demagnetising field and thus the internal field change. Although this effect can be negligible at extremely high fields or for materials with a low magnetisation and anisotropy, for moderately high fields (below $1600 \mathrm{kA} / \mathrm{m}$ ) this effect can be considerable, with demagnetising fields of the order of several hundreds of $\mathrm{kA} / \mathrm{m}$; this influences in particular the hysteresis loss measurements. Therefore compensation for demagnetising fields should be applied during the measurement. We have used the method proposed by Bernards et al. [3], in which the internal field value and direction are kept constant during the measurement by varying the magnitude of the applied field and its direction. Using this method, ADR and torque measurements have been performed. In the following, these compensated measure- 


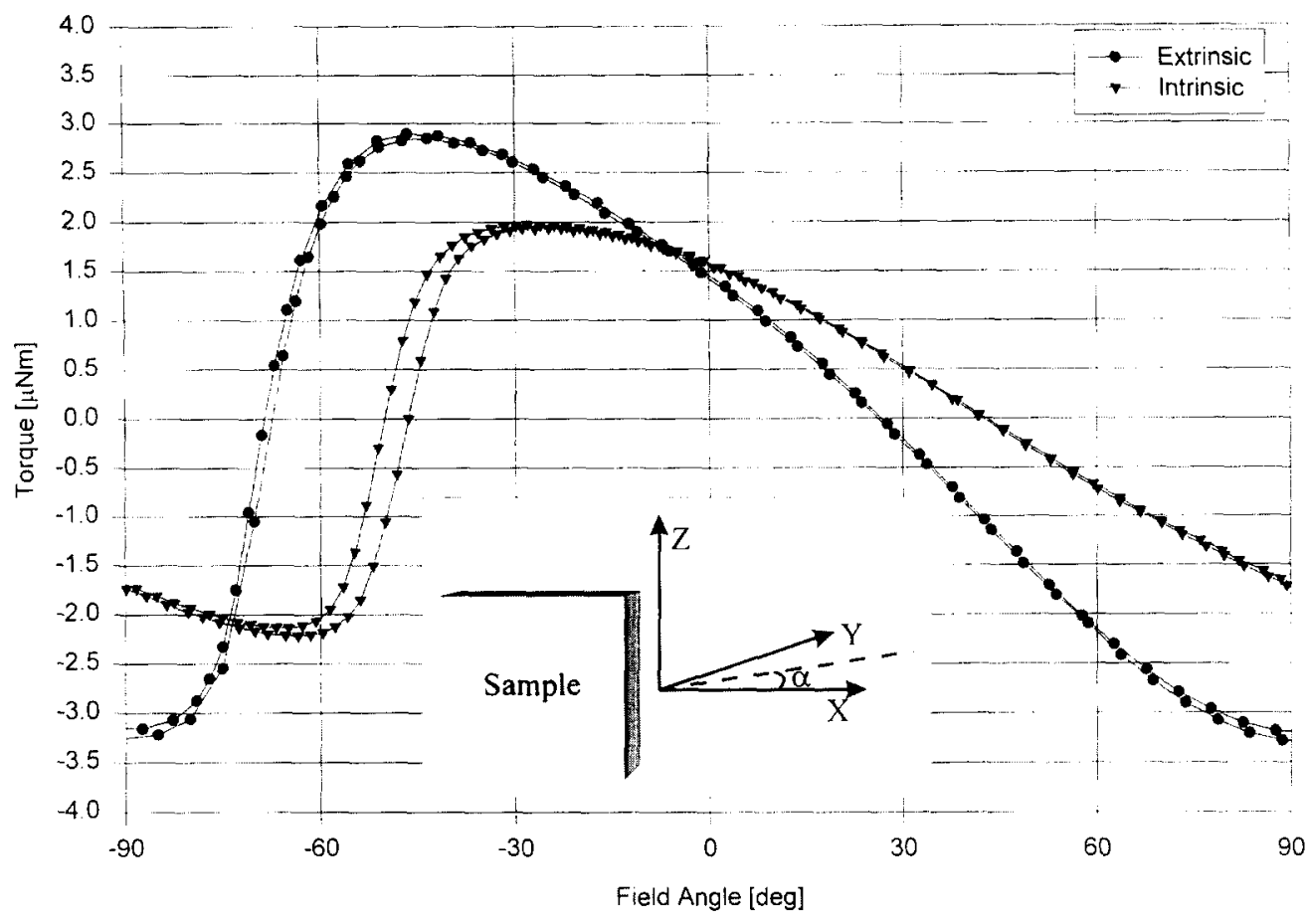

Fig. 1. Extrinsic and intrinsic torque curves performed on a Sony single-layer ME tape. $0^{\circ}$ is parallel to the sample plane. The difference in the maximum torque values results from the absence of the demagnetisation torque. The inset shows the coordinate system used. The field is applied in the $x-y$ plane. The z component of the magnetisation can be neglected, since the magnetisation vector will always remain in the plane defined by the field and the (effective) anisotropy direction (the $x-y$ plane).

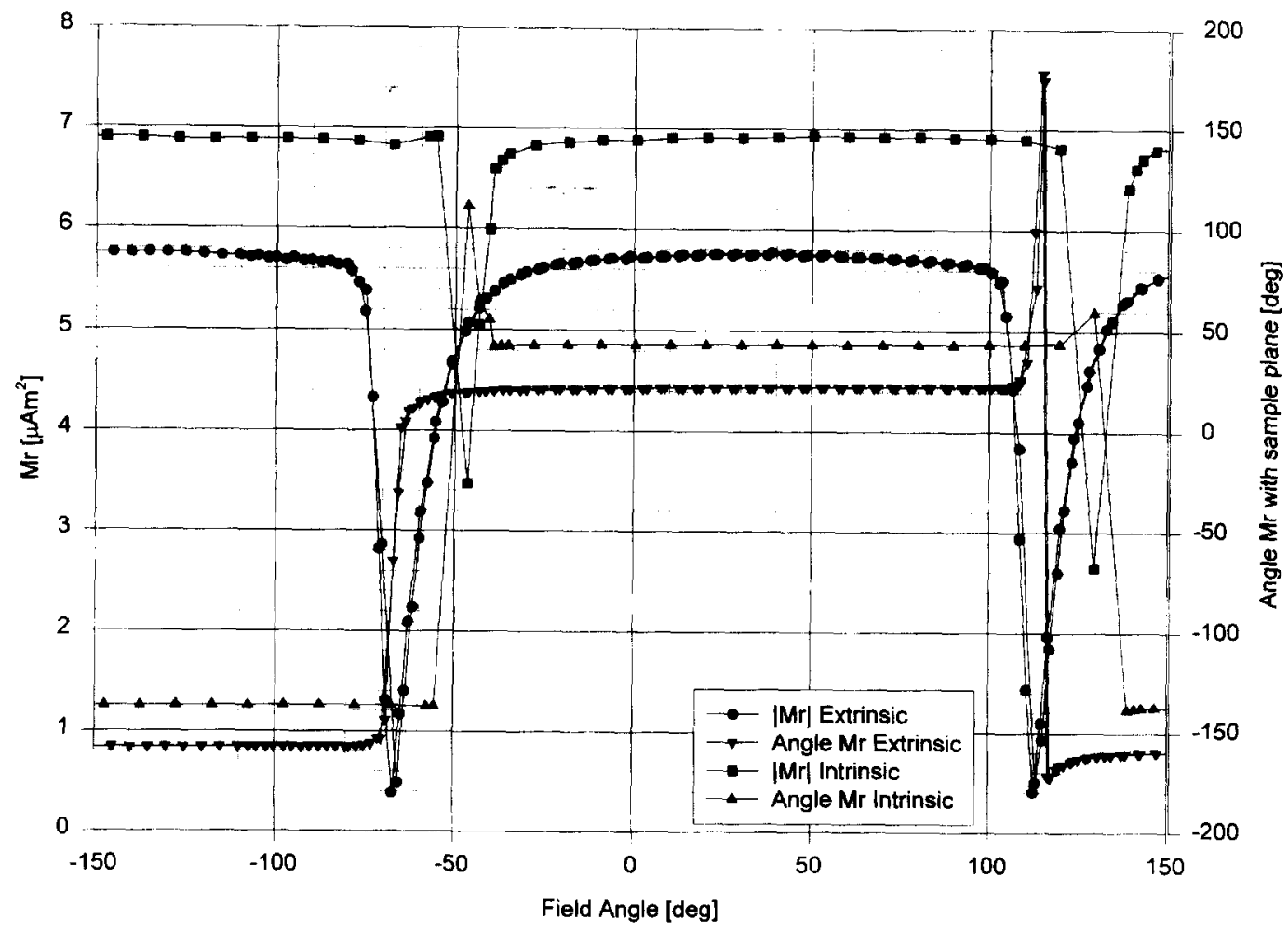

Fig. 2. Intrinsic and extrinsic angle-dependent remanence measurements on the ME tape from Fig. 2. 
ments are referred to as 'intrinsic', in contrast to the 'extrinsic' uncompensated measurements.

\section{Experimental results}

In Fig. 1 we present the extrinsic torque curve for a Sony HI-8 ME tape, which shows an easy-axis orientation of $26^{\circ}$, whereas the intrinsic measurement results in an easy axis direction of $43^{\circ}$, which is approximately equal to the column orientation as observed in TEM micrographs.

If a standard torque curve is corrected for the demagnetising field after the measurement (by correcting for the contribution of $\frac{1}{2} \mu_{0} M_{\mathrm{s}}^{2}$ ), roughly the same easy axis will be found. However, one has to keep in mind that at lower fields this method is not valid because the change in the internal field becomes too large. When torque measurements are used to determine the hysteresis loss, this effect should be taken fully into account.

ADR measurements can only be corrected during the measurements. The intrinsic and extrinsic ADR measurements on the sample of Fig. 1 are shown in Fig. 2. These results are fairly easy to interpret; one can see that the remanent magnetisation always has a direction of $22 \pm 180^{\circ}$ with the plane of the film $(x)$. Another way of retrieving the easy axis from an ADR measurement is to look at the point of minimum remanence, which is a hard axis. This leads to an easy axis of $24^{\circ}$. We attribute the discrepancy between these and the torque values to the non-saturated state during the torque measurement. Torque measurements at much higher fields (in a torque magnetometer) indicate an easy axis of $22^{\circ}$.

In a similar way the intrinsic easy axis can be found from Fig. 2, which is at $44 \pm 180^{\circ}$, approximately equal to the value found from the intrinsic torque measurement.

\section{Bilayer media}

ADR measurements on bilayer media provide information on the resulting easy axes, but they are much more difficult to interpret. Fig. 3 shows the extrinsic and intrinsic ADR measurements performed on a commercial bilayer (TDK) ME tape. The inset shows the two possibilities for adding the magnetisation vectors of the individual layers.

From these measurements we see that the vectors add up to a vector with a length of $5.334 \mu \mathrm{A} \mathrm{m} \mathrm{m}^{2}$ at $0^{\circ}$, and a vector with a length of $2.25 \mu \mathrm{A} \mathrm{m}^{2}$ at $105^{\circ}$. Assuming no interaction between the layers, for situation 1 we can write $M_{\text {total } . x}=5.334, M_{\text {total }, y}=0$.

For situation 2 we can write

$M_{\text {total }, x}=2.25 \cos (105), M_{\text {total }, y}=2.25 \sin (105)$.

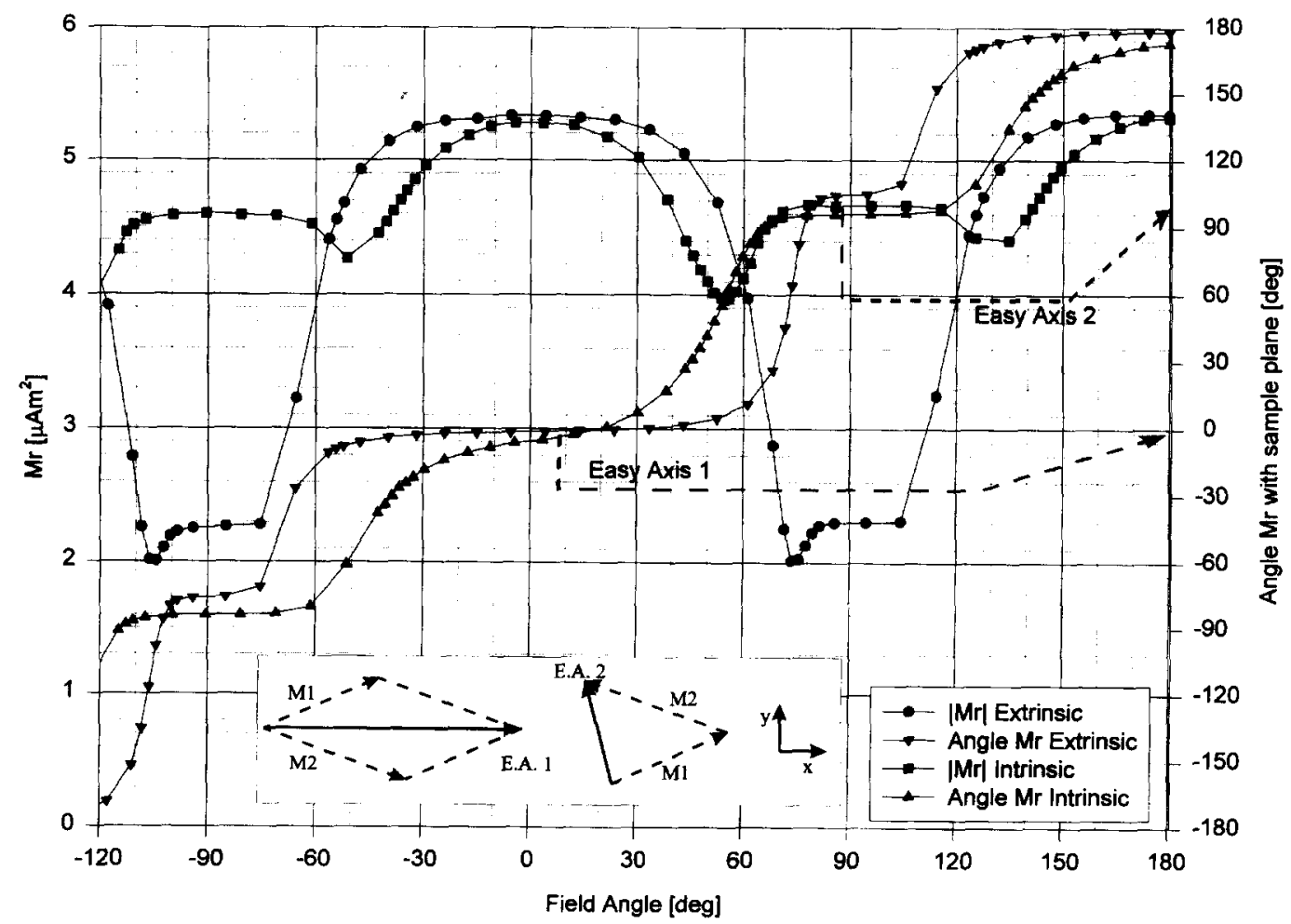

Fig. 3. Intrinsic and extrinsic ADR measurements on a commercial bilayer TDK ME sample. The inset shows how the vectors of the individual layers add up to the global easy axes; the extrinsic easy axes are indicated by dashed arrows. 
This leads to

$M_{1, x}+M_{2, x}=5.334, M_{1, x}-M_{2, x}=-0.5823$,

$M_{1, y}-M_{2, y}=0, M_{1, y}+M_{2, y}=2.173$.

From this we can calculate

$M_{\text {।. }}=2.376, M_{1, y}=1.087$,

$M_{2 . .1}=2.958, M_{2 . r}=1.087$,

which can also be represented as vectors with lengths $\sim 2.6$ and $\sim 3.2 \mu \mathrm{A} \mathrm{m}^{2}$ at angles of $\sim 24.6^{\circ}$ and $\sim 20.2^{\circ}$, respectively. Similar reasoning leads to intrinsic vectors with lengths $\sim 3.15$ and $\sim 3.87 \mu \mathrm{A} \mathrm{m}{ }^{2}$ at angles of $40.8^{\circ}$ and $\sim 42.1^{\circ}$, respectively.

The assumption of non-interacting layers is questionable, however. This becomes clear when looking at Fig. 4, which shows extrinsic ADR measurements on a doublelayer tape produced on a mini-roll coater system [4] (the sample is considered in more detail as sample B in Ref. [5]).

In Fig. 4 we show a comparison of the ADR measurements on the double-layer tape and the summation of the results obtained from the individual layers. From this we see that the minima obtained at the angles at which the remanence of the top layer is minimal, are approximately equal for the two curves. For other angles, the mutual influences between the layers lead to a higher remanence for the double-layer tape than could have been expected from the results of the individual layers. We attribute this to an aligning effect of the magnetisation direction in each layer caused by the stray field of the other layer. Immink et al. [5] have also observed interactions between the layers of the same sample.

The top layer of the double layer is evaporated onto another ME layer, which might cause a different structure when compared with single layers evaporated onto (much smoother) PET substrates. Therefore (part of) the observed differences might be attributed to structural differences as well. It is therefore difficult to draw conclusions from these results.

\section{Conclusions}

Torque and ADR measurements are strongly influenced by the demagnetising field. Demagnetisation compensation produces information on the intrinsic easy axis of media with oblique anisotropy directions. For ADR measurements in particular, this presents interesting information which is in agreement with observations of the morphology and microstructure of the media. ADR measurements from double-layer media are more difficult to analyse and interpret. Differences between the measurement results of double-layer media and the added results of the individual layers might indicate magnetostatic interactions between the layers.

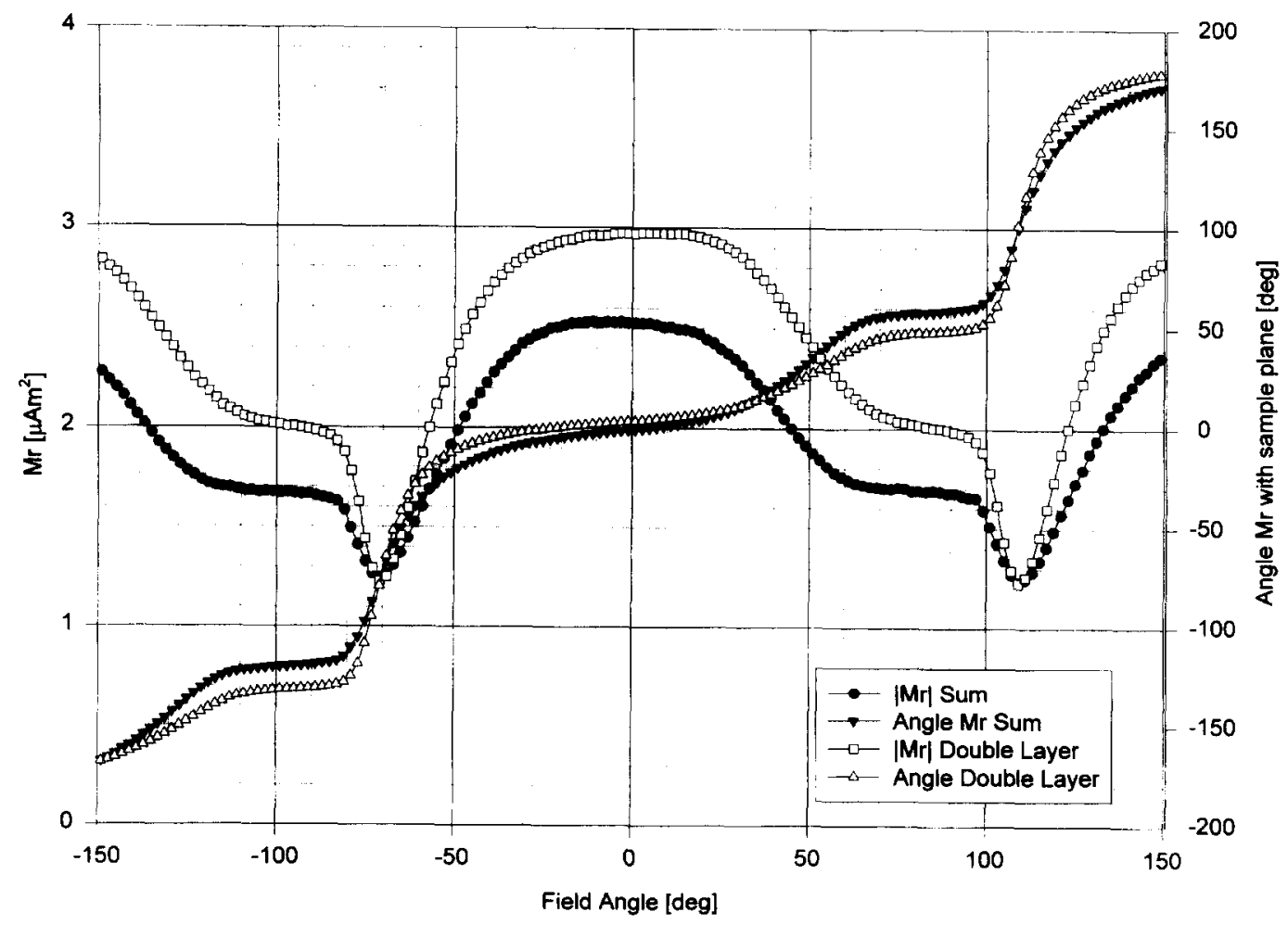

Fig. 4. Extrinsic ADR measurements on sample B from Ref. [5], compared with the added results from the individual layers. 


\section{Acknowledgements}

The authors wish to thank Oxford Instruments for financial support.

\section{References}

[1] L. Abelmann, V. Kambersky, J.C. Lodder and Th.J.A. Popma, IEEE Trans. Magn. 29 (1993) 3022.
[2] H.J. Richter and H. Hibst, J. Magn. Magn. Mater. 95 (1991) 118.

[3] J.P.C. Bernards and H.A.J. Cramer, IEEE Trans. Magn. 26 (1991) 4873.

[4] L. Abelmann, J.C. Lodder and Th.J.A. Popma, J. Magn, Soc. Jpn. 18, Suppl. S1 (1994) 295.

[5] A.H.J. Immink, M.D. Bijker, L. Abelmann and J.C. Lodder, J. Magn. Magn. Mater. 155 (1996) 279 (these Proceedings). 